\title{
Microwaves: thin metal slits and liquid crystals.
}

\author{
J Roy Sambles ${ }^{1}$, Alastair P Hibbins ${ }^{1}$, Robert J Kelly ${ }^{1}$, James R Suckling ${ }^{1}$ and Fuzi Yang ${ }^{2}$ \\ ${ }^{1}$ Thin Film Photonics Group, School of Physics, University of Exeter \\ ${ }^{2}$ Chemistry Department, Tsignhua University, Beijing 100084, P.R. China
}

\begin{abstract}
Using liquid crystals to control the propagation of microwaves is a potentially interesting technology. By incorporating small amounts of liquid crystal in thin slat metal structures through which the microwaves may resonantly pass a whole new range of voltage tuned microwave devices are becoming available. Metallic sub-wavelength slit structures at microwave frequencies have been constructed which show Fabry-Perot type resonances in very thin slits. If the dielectric in such thin slits is an aligned liquid crystal it is found possible to voltage-control the resonant frequencies. Novel selective filters and structures for microwave beam steering have been fabricated leading to a new generation of liquid crystal controlled devices.
\end{abstract}

Keywords: Transmission, sub-wavelength aperture, slit, microwave, resonant filters

\section{INTRODUCTION}

\subsection{Previous work}

\subsubsection{Microwaves and liquid crystals}

We shall ignore strip line devices, which allow liquid crystals to be easily made part of the structure but which have many other limitations. One of the earlier 'bulk' devices to employ microwaves with liquid crystals was demonstrated by Lim et al ${ }^{1,2}$ who proposed a millimetre wave phase shifter. Birefringence of the liquid crystal material at microwave frequencies was measured by containing it within a section of rectangular waveguide, and placing it in one arm of an interferometer. The phase shift produced by the liquid crystal was compensated for by a $360^{\circ}$ mechanical phase shifter in the other arm of the interferometer. To obtain a well aligned monodomain a magnetic field of $5 \mathrm{kG}$ was imposed by a permanent magnet. The director alignment (average orientation of the molecular axes) was parallel to the $\boldsymbol{E}$-vector of the millimetre waves. A central plane electrode placed within the waveguide, parallel to the long sides of the waveguide, 
allowed application of a modulating electric field to the liquid crystal material. As this electric field was increased above a threshold voltage of $60 \mathrm{~V}$, there was a clear increase in phase shift with increasing voltage, until saturation at about $200 \mathrm{~V}$ where the liquid crystal was completely re-aligned with the electric field. The response time of the liquid crystal alignment to the electric field was about $0.1 \mathrm{~s}$, with a relaxation time under the magnetic field of about $2 \mathrm{~s}$.

Further work has been conducted on millimetre-wave phase shifters using liquid crystal waveguides by Guerin et al $(1997)^{3}$. Tanaka et al (2000) $)^{4}$ investigated the transmission properties of a grating-patterned electrode structure LC cell. The transmittance of the LC cell was found to be $70-80 \%$ for polarization parallel to the grating vector, and $7-8 \%$ for polarization perpendicular to the grating vector. It was found that changing the orientation of the liquid crystal director via an applied electric field had an effect on the transmissive properties, even though the field was inhomogeneous due to the grating pattern of the electrode structure. Later work by Tanaka and Sato $(2001)^{5}$ concerned an LC prism, consisting of a wedge-shaped stack layered structure of metal substrates and nematic liquid crystal dielectric material, as shown below. The millimetre wave polarization direction was parallel to the grating vector of the stack-layered structure. Metal slats allow the application of voltages to change the alignment of the liquid crystal thereby changing the angle of deflection of the millimetre wave.

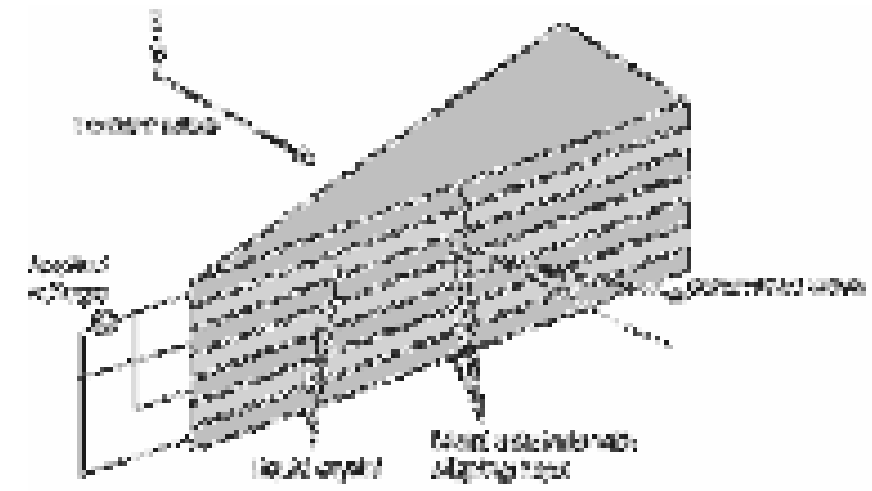

Figure 1. Geometry used by Tanaka and $\mathrm{Sato}^{4}$ for the liquid crystal prism device.

Tanaka and Sato, (2002) ${ }^{6}$ subsequently devised a liquid crystal lens based on the same principles, now using a set of planoconvex metal plates as electrode substrates. The stack of metal plates and liquid crystal layers were as shown in figure 2, with the liquid crystal layer thickness dictated by glass ball spacers. To facilitate alignment of the liquid crystal the metal substrates were coated with an aligning layer of polyvinyl alcohol which was unidirectionally rubbed. As before the polarisation of the millimetre wave was parallel to that of the grating vector of the stack. At zero volts the director is aligned parallel to the substrate (homogeneous) due to the aligning layer, and the change in focal length occurs when a voltage is applied across the LC layers, which causes the director to align along the direction of the applied field, changing the effective refractive index of the stack. A focal point of about $78 \mathrm{~mm}$ was found with no voltage applied, and this changed to about $62 \mathrm{~mm}$ due to the application of a $10 \mathrm{~V}$ electric field. 


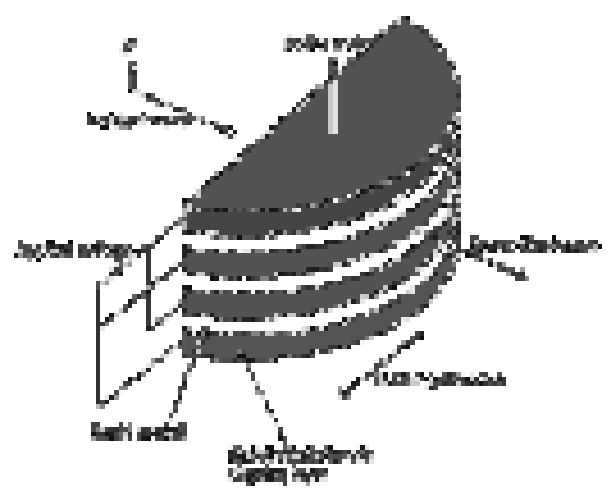

Figure 2. Liquid crystal microwave lens fabricated by Tanaka and Sato ${ }^{6}$.

Fujikake et al $(2001)^{7}$ demonstrated the possibility of using a fine polymer network to stabilize the director within a thick liquid crystal cell, by applying a photopolymerization-induced phase separation technique. This technique was applied in a phase shifting device with a $100 \mu \mathrm{m}$ thick LC layer. It resulted in a phase shifter that achieved similar results to previous devices but with a faster relaxation time due to the polymer network.

Two of the limiting factors of devices discussed so far are slow response times and high insertion loss. These issues were addressed by Kuki at al (2002) $)^{8}$ in the construction of a variable delay line. A dual-frequency switching-mode liquid crystal (DFSM LC) was used. The advantage of DFSM LC is that the alignment under the application of an electric field depends on the frequency of the control voltage. The DFSM LC used in this experiment aligns parallel to the field when the control voltage is in the low frequency region, near dc, whereas in the higher frequency region of tens of kilohertz the director aligns perpendicular to the direction of the applied field. This means that the liquid crystal director can be driven into either parallel or perpendicular alignment, rather than having to relax into one alignment from the other, reducing response time. The voltage was applied via a strip conductor, which had a narrow width in order to keep the characteristic impedance constant but which lead to substantial insertion loss.

Tanaka and co-workers appear to be the first to appreciate that thin metal sub-wavelength slat structures may be the way forward for liquid crystal devices for microwave applications. We now turn our attention to the transmitting properties of such slat structures before looking in more detail at recent developments with metal slit structures and liquid crystals.

\subsubsection{Thin metal slit structures and microwaves}

The observation by Ebbesen and coworkers ${ }^{9}$ of remarkably enhanced transmission of radiation through sub-wavelength holes in silver films has caused an explosion of interest, both theoretical ${ }^{10}$ and experimental in this phenomenon. Their results were for small holes in metal films and it should be emphasised that this is a very different geometry to that of 
narrow slits, sometimes wrongly used to model those first results. Nevertheless sub-wavelength slit systems do provide an interesting structure in their own right even though they support (as noted by Porto et al ${ }^{11}$ ) a family of TEM mode standing wave states through the metal film. This is in contrast with sub-wavelength holes which support no TEM mode, and so no such states.

At microwave frequencies it is rather straightforward to construct sub-wavelength slit structures and to test their electromagnetic response. Further the electromagnetic absorbance by the metal due to its finite conductivity is also then expected to have little effect, although, as we shall see for very thin slits, this is a flawed assumption. Studies ${ }^{12}$ of subwavelength slat structures (zero order gratings) show the expected 'filled' Fabry-Perot behaviour. Further work ${ }^{13}$ took this to two dimensions using a 'wall' of metallic bricks which gave remarkable transmission of microwaves of any polarisation.

All the above studies tended to use slits which, although less than half a wavelength wide, were generally beyond 250 $\mu \mathrm{m}$. With microwave wavelengths being around $10 \mathrm{~mm}$ these slits still satisfy the sub-wavelength condition but are certainly not thin enough for the purposes of aligning liquid crystals, where typically cell thicknesses of order $10 \mu \mathrm{m}$ are used. This small thickness is required simply to allow good alignment of the liquid crystal director, which demands a cell thickness less than of order $100 \mu \mathrm{m}$ unless external aligning fields are applied. In order to explore the use of voltage-controlled (as opposed to cumbersome magnetic-field control) liquid crystals in microwave devices it is therefore essential to explore first the propagation of microwaves through much thinner slits. Once the behaviour of such metal slit structures has been established then filling them with aligned liquid crystals should not be too much of a problem.

\subsection{Theory}

\subsubsection{Single metal-dielectric interface}

The interaction of electromagnetic radiation with metals inevitably involves the potential for the excitation of surface plasmons. These are surface charge density oscillations together with their associated electromagnetic fields. Their dispersion relation is readily obtained by appreciating that they are really none other than modes which satisfy the Brewster condition. Consider a plane interface between two dielectrics. If the incident medium is described by refractive index $n_{1}$ (relative permittivity $\varepsilon_{1}=n_{1}{ }^{2}$ ) with angle of incidence $\theta_{1}$ and the second medium has index $n_{2}\left(\varepsilon_{2}=n_{2}{ }^{2}\right)$ then for light polarised with its electric vector lying in the plane of incidence (p-polarised or Transverse Magnetic) there is a simple Brewster condition, when there is no reflected component, given by $\tan \theta_{\mathrm{B}}=n_{2} / n_{1}=\left(\varepsilon_{2} / \varepsilon_{1}\right)^{1 / 2}$. This is very well known. This expression may be rewritten to solve for the in-plane component of the incident momentum $n_{1} \hbar k \sin \theta_{\mathrm{B}}$ leading to $n_{1} \hbar k \sin \theta_{\mathrm{B}}=n_{1} \hbar k\left(\tan ^{2} \theta_{\mathrm{B}} /\left(1+\tan ^{2} \theta_{\mathrm{B}}\right)\right)^{1 / 2}=n_{1} \hbar k\left(\varepsilon_{2} /\left(\varepsilon_{1}+\varepsilon_{2}\right)\right)^{1 / 2}$. For a trapped surface wave, the surface plasmon, this leads to the very simple expression for the in plane wavevector of the surface plasmon as $\hbar k_{\mathrm{SP}}=\hbar k\left(\varepsilon_{1} \varepsilon_{2} /\left(\varepsilon_{1}+\varepsilon_{2}\right)\right)^{1 / 2}$. 
All that is required is that this corresponds to a Brewster angle beyond $90^{\circ}$ since then there is no real propagating wave solution. For this to be the case then $\varepsilon_{2}$ has to be negative and have magnitude greater than $\varepsilon_{1}$. Metals satisfy this condition readily for frequencies below the surface plasmon cut-off (which is generally in the ultra-violet). In the microwave domain where the real part of the metallic permittivity is very negative (typically $-10^{3}$ to $-10^{4}$ ) the surface plasmon momentum is very close to that of a polariton in the adjacent dielectric. Thus, as illustrated in figure 3 the surface plasmon is very close to the light line and it is almost a plane wave trapped at the metal surface. There will be a small penetration of fields into the metal (the skin depth of order microns) and a very long penetration depth (almost a plane wave) into the dielectric.

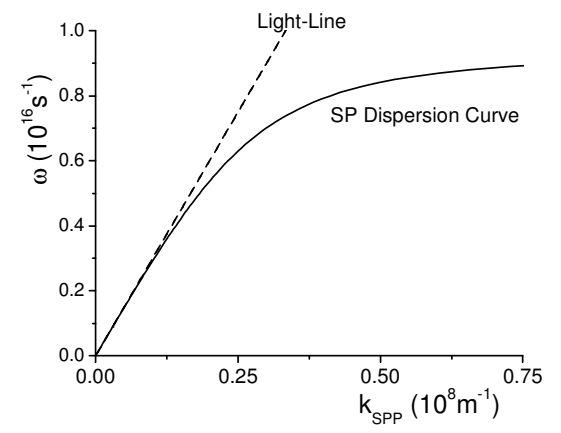

Figure 3. Schematic of the surface plasmon dispersion curve. For microwave frequencies, at the bottom left corner, the light line and surface plasmon curves almost coincide.

It is important to introduce these ideas at this stage as for the large part researchers assume that at microwave frequencies metals may be treated as perfect conductors leading to zero skin depth and simple plane waves at the surface. As we shall see later this is not the case and this matters significantly for electromagnetic waves confined within thin slits in a metal.

\subsubsection{Double metal dielectric interface, slit modes, or coupled surface plasmons}

Thus far we have considered only the surface plasmon at a single metal-dielectric interface but the essence of this study is microwaves confined in thin slits in a metal plate. Thus we need to examine the dispersion relation for modes associated with two adjacent parallel metal surfaces. This is a well explored problem ${ }^{14}$ and the dispersion equation for the coupled surface plasmon modes is given by the lowest order solution to:

$$
\tan \left(\frac{\alpha_{1} w}{2}\right)=-\frac{\varepsilon_{1} \alpha_{2}}{\varepsilon_{2} \alpha_{1}}
$$

where $\alpha_{j}^{2}=k^{2}-\varepsilon_{j} k_{0}^{2}$, with $k_{0}$ the free space wavevector. In the situation we are here concerned with the slit width $w$ is much less than the wavelength. Thus we may approximate equation (1) to: 


$$
\frac{\alpha_{1} w}{2} \approx-\frac{\varepsilon_{1} \alpha_{2}}{\varepsilon_{2} \alpha_{1}}
$$

Now at microwave frequencies the metal permittivity is given approximately by $\varepsilon_{2}=i \sigma_{0} / \varepsilon_{0} \omega=\varepsilon_{r}-i \varepsilon_{i}$, where $\sigma_{0}$ is the conductivity. Then taking $\varepsilon_{1}=1$ (air), $\alpha_{2}^{2}=k^{2}-\varepsilon_{2} k_{0}^{2} \approx k^{2}+i \varepsilon_{i} k_{0}^{2} \approx i \varepsilon_{i} k_{0}^{2}$ equation (2) reduces to:

$$
\alpha_{1}^{2} w \approx \frac{2 \sqrt{i}\left|\varepsilon_{i}\right|^{1 / 2} k_{0}}{-i\left|\varepsilon_{i}\right|} \approx \frac{2 k_{0}}{\left|\varepsilon_{i}\right|^{1 / 2}} e^{i 3 \pi / 4}
$$

In turn, since $\alpha_{1}^{2}=k^{2}-k_{0}^{2}$, this leads to:

$$
k^{2} \approx k_{0}^{2}+\frac{2 k_{0}}{\left|\varepsilon_{i}\right|^{1 / 2} w} e^{i 3 \pi / 4}
$$

Thus the lowest order (closest to plane wave) coupled mode which exists between two parallel metal plates spaced small distance apart has its wavevector modified from $k_{0}$ by the finite conductivity of the metal. With $\varepsilon_{i}$ of order $10^{7}$ and $k_{0}$ of order $600 \mathrm{~m}^{-1}$ then for a $w$ of $100 \mu \mathrm{m}$ this correction term in equation (4) is $\sim 1 \%$ of $k_{0}^{2}$, which is not very significant. However for thinner slits, which are better for aligning liquid crystals, $w$ may be $10 \mu \mathrm{m}$. In which case this correction term is of order $10 \%$, tending of course to dominate for impossibly thin slits. Thus the finite conductance will have a significant effect on both the wavevector of the modes in the thin slits, and also because of the complex part of equation (4) the width of any resonances. (This influence of finite conductivity we label the Preist-Sambles effect ${ }^{15}$.)

\subsubsection{A physical interpretation of the conductivity effect}

While the mathematics above is relatively straightforward it is worthwhile exploring what is causing the wavevector shift. If we consider the boundary conditions at the metal walls we see that there will always have to be finite field curvature to satisfy both the field amplitude and its gradient. Normally the conductivity is so high that the skin depth in the metal is negligible relative to the dimensions of the problem (wavelength or slit width). Then it is supposed that the boundary condition leaves a simple plane wave solution in the slit. Thus $\alpha_{1}^{2}$ is zero, or the electric field orthogonal to the slit walls is simply invariant across the slit. However as $w$ reduces $\alpha_{1}^{2}$ becomes negative. That is the wavevector component perpendicular to the walls has an exponential character beginning to be imposed upon it. This is perhaps best understood by examining the normal component of $\mathbf{D}$. This has to be continuous, as does its gradient across the air/metal boundary. Consequently near the walls there has to be a spatial gradient to match to the gradient inside the metal, due to the skin effect. Thus there will be, over a distance in the air of order a few skin depths, a spatially varying D field. This variation, which is exponential rather than sinusoidal in character, gives rise to the change in the wavevector in the orthogonal direction. At microwaves since the skin depth is of order $1 \mu \mathrm{m}$ it is only when the slit width becomes of this order that significant changes are expected. Thus for metal slits, because the surface plasmon at microwave frequencies has very nearly the same wavevector as a free photon the lowest order coupled modes allowed to exist in the slits are essentially little changed from plane free photons. 


\section{RESULTS}

\subsection{Microwaves and metal slits}

\subsubsection{Single slits}

\subsubsection{Without liquid crystal}

The simplest slit geometry is shown in figure $4 \mathrm{a}$.
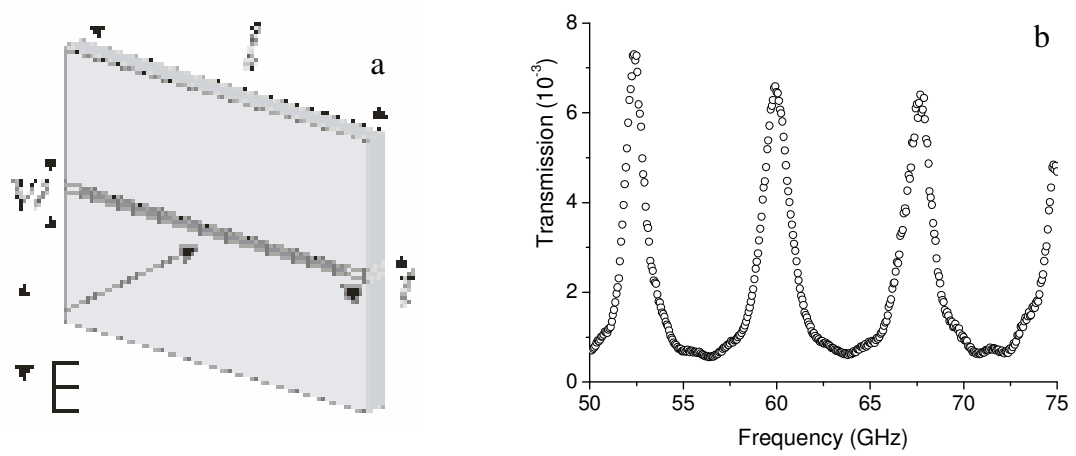

Figure 4. a. Geometry to be explored for resonant transmission of a single variable width slit in an aluminium plate of thickness t. The microwave field is polarised orthogonal to the slit and incident normally on to the metal plate. b. Typical transmissivity scan for a slit width of $250 \mu \mathrm{m}$.

Using a collimated microwave beam the transmissivity of such a structure is monitored as the frequency of the source is varied. This gives a series of resonant peaks with frequencies closely specified by the simple Fabry-Perot expression:

$$
f=\frac{c N}{2 t n}
$$

where $N$ is an integer, $n$ is the refractive index of the dielectric between the metal plates and c is the velocity of light.

Detailed studies of the effect of changing slit width on these resonant modes have already been undertaken ${ }^{15}$. These confirm that there is indeed a strong influence of the finite conductance of the metal as the gap thickness is reduced below about $80 \mu \mathrm{m}$. Further they show that the boundary conditions on the fields at the entrance and exit apertures also influence the resonant frequencies. The wider the slits the more the resonant frequency is reduced. While this accords with the trend of an analytic formulation for end effects given by Takakura ${ }^{16}$, the absolute magnitude does not. However there is full agreement with the predictions of finite element modeling code ${ }^{17}$ using the conductivity of the metal as fitting parameter. This study clearly confirms that a single slit in a metal plate gives resonant transmission due to the 
excitation of Fabry-Perot like resonances. These electromagnetic states inside the slits are almost plane wavelike in character with a wavelength very close to that of free space photons. Hence they closely match in frequency the predictions of equation (5), which for figure 5a would correspond to $68.7 \mathrm{GHz}$. However, as mentioned, there is a significant and readily measured shift from this prediction due to two effects. The first, associated with end effects, is a reduction in resonant frequency at high slit widths, the second a reduction at low slit widths due to the finite conductance of the metal.
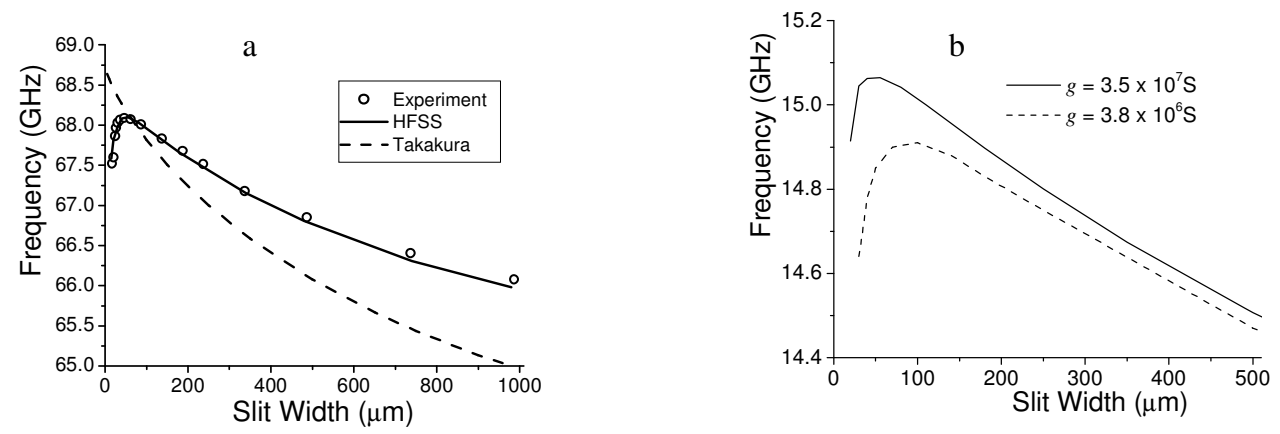

Figure 5. a. Frequencies of the resonances (corrected by $-0.035 \pm 0.008 \mathrm{GHz}$ for beam width effects) for one of the Fabry-Perot modes as a function of slit width. Data (circles) is compared with finite element modeling using HFSS $^{17}$ software with a conductivity of 1.7 $\mathrm{x} 10^{7} \mathrm{Sm}^{-1}$ (solid line) and Takakura ${ }^{16}$ prediction (dashed line). b. HFSS predictions for the lowest resonance recorded, showing the influence of a significant decrease in conductivity, $\mathrm{g}$, on the decrease in resonant frequency for small slits.

These results show that in the frequency band from 20 to $70 \mathrm{GHz}$ this reduction in frequency at small slit widths occurs below about $100 \mu \mathrm{m}$. Figure 5b illustrates the influence of a significantly reduced metal conductivity on the turn down in resonant frequency of the longest wavelength mode observed with this system $(\mathrm{N}=2)$.

\subsubsection{With a liquid crystal}

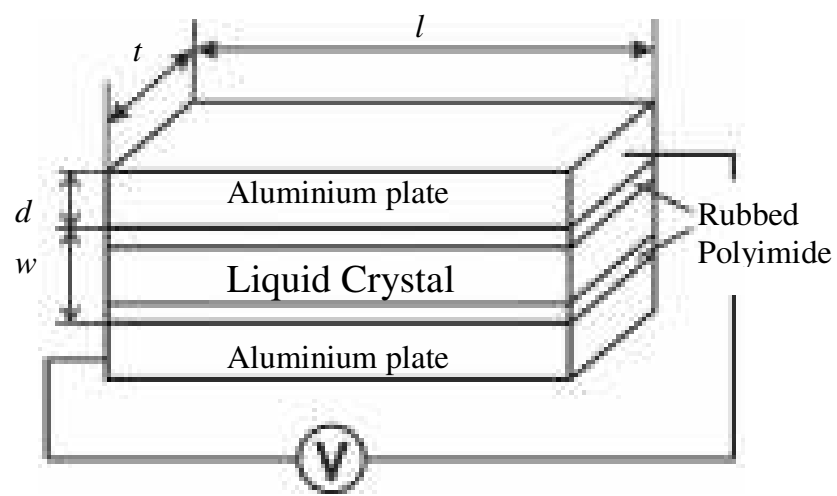

Figure 6. Schematic of single slit filled with liquid crystal to give voltage-tuned microwave transmission 
A very narrow single metallic slit is built from a pair of aluminium plates (see figure 6) with insulating mylar spacers at each short end ${ }^{18}$. The dimensions of the plates are length $l=60.00 \mathrm{~mm}$, width $t=20.00 \mathrm{~mm}$ and depth $3.00 \mathrm{~mm}$. To facilitate alignment of the liquid crystal the two inner surfaces of the aluminium plates, the walls of the slit cavity, are polished to mirror quality and spin-coated with a polyimide. They are then baked and uni-directionally rubbed along the short edge direction of the plates to provide homogeneous (planar) alignment of the liquid crystal. The polyimide layers also prevent ions entering the thin nematic layer when a field is applied. These two treated surfaces are finally placed together face-to-face with the $75 \mu \mathrm{m}$ mylar spacers separating them. This structure is then capillary filled with nematic liquid crystal (E7, Merck-BDH) and the plates are connected to an AC voltage source $(10 \mathrm{kHz})$. This single metallic slit sample is then inserted in an absorber aperture and examined for its microwave transmission properties.

Transmission data were taken as a function of frequency and also of applied voltage across the liquid-crystal-filled slit. As expected there is a set of resonant transmission peaks which move in frequency when various voltages are applied between the plates. Figure 7a shows typical frequency-dependent and voltage-dependent transmission spectra.

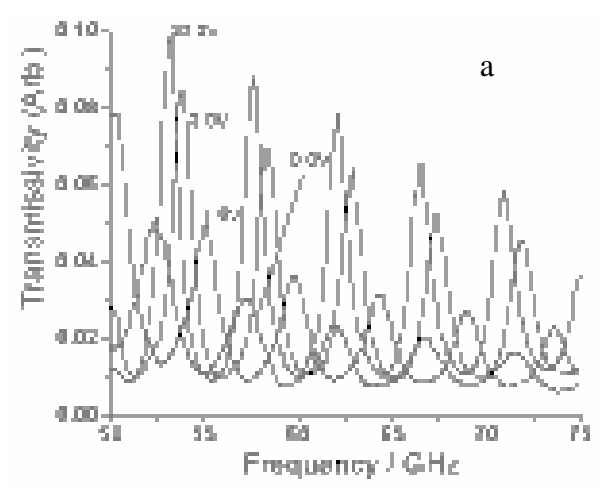

$1:$

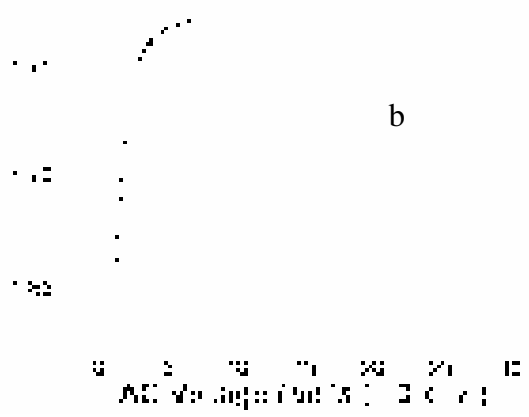

Figure 7. a. Transmissivity response of a single slit between two metal plates, filled with aligned nematic liquid crystal. b. Voltage dependence of the effective refractive index of the liquid crystal deduced from the resonant transmission peaks.

Data were taken for voltages $(10 \mathrm{kHz})$ from $0.0 \mathrm{~V}$ to $30.0 \mathrm{~V}(\mathrm{rms})$. The peaks move down in frequency as the voltage is increased. This movement corresponds to increased external wavelength fitting into the length of the slit due to increased refractive index inside the slit. With the electric field of the incident radiation across the slit this indicates that the originally homogeneously aligned (parallel to slit walls) liquid crystal is beginning to become homeotropically aligned (perpendicular to slit walls) as the voltage is applied. One mode step in frequency ( $4 \mathrm{GHz})$ is completely encompassed by scanning over this range of voltage. The greatest change in mode position for a given change in voltage occurs between $1.0 \mathrm{~V}$ and $3.0 \mathrm{~V}$. If the mode positions from the recorded data are introduced into the simple Fabry-Perot equation (5) then, ignoring end corrections, the mode order numbers are $N=11,12,13,14$ and 15 respectively (at zero volts, beginning at the lowest frequency mode in figure 7a). No significant index dispersion of the material over this 
frequency region is found. By fitting equation (5) to the transmissivity peaks the voltage dependent refractive index is found, as shown in figure $7 \mathrm{~b}$.

From figure $7 \mathrm{~b}$ we note clearly the rapid change of index with voltage between $1.0 \mathrm{~V}$ and $3.0 \mathrm{~V}$ but after this the change appears to saturate. This is because at these higher voltages the liquid crystal director is almost completely homeotropically aligned throughout the slit. (Note that only of order $5 \mathrm{~V}$ is required to almost completely switch the structure, fully compatible with semiconductor technology drive voltages.) From the data of figure $7 \mathrm{~b}$ we may deduce that the indices obtained at $0.0 \mathrm{~V}, n=1.654$, and at $30.0 \mathrm{~V}, n=1.780$, are effectively the ordinary and extraordinary indices of E7, $n_{\mathrm{o}}$ and $n_{\mathrm{e}}$, in this frequency region. Then the anisotropy of E7 is $\Delta n \approx 0.13$ for 50.0-75.0 GHz. This also accords well with the result of Lim et $\mathrm{al}^{2}$. For intermediate voltages the situation is a little more complex. Now the director tends to homeotropic alignment at the centre of the slit while remaining homogeneous at the walls. Thus there is a distribution of refractive index across the slit. However because $w / \lambda$ is very small the microwave field distribution may be treated as quasi-uniform. The effective index for each mode is then, to first order, the average calculated from the spatial profile of the director field. Continuum elastic theory ${ }^{19}$ is used to model the tilt angle, $\theta$, distribution with the parameters of E7: $\varepsilon_{\|}=19.50, \varepsilon_{\perp}=5.40, \mathrm{k}_{11}=1.15 \times 10^{-11} \mathrm{~N}$ and $\mathrm{k}_{33}=1.46 \times 10^{-11} \mathrm{~N}$. From this the effective local index is calculated using:

$$
n_{\mathrm{eff}}=n_{\mathrm{o}} n_{\mathrm{e}} /\left(n_{\mathrm{e}}{ }^{2} \cos ^{2} \theta+n_{\mathrm{o}}{ }^{2} \sin ^{2} \theta\right)^{1 / 2}
$$

with $n_{\mathrm{e}}=1.782$ and $n_{\mathrm{o}}=1.654$ at these frequencies. Then finally by integrating across the liquid crystal layer we find the average effective index as a function of applied field. This is shown as the solid line in figure $7 \mathrm{~b}$. The agreement between the solid line and data is very good. This experiment then establishes the principle of using well aligned liquid crystal in a small slit in a metal plate to control microwave transmission. (We can of course also use this simple geometry to measure the microwave properties of small amounts of novel materials ${ }^{20}$.) Other more elaborate slat structures may now be considered.

\subsubsection{Multiple slits}

\subsubsection{Without liquid crystal}

The resonant transmission of microwaves through a stack of metal plates is well known ${ }^{12}$. Provided the stack is spaced such that it is non-diffractive over the wavelength range studied then it responds very similarly to a single slit but with much higher transmissivity and much less spreading of the exit beam. It becomes in effect a 'filled' Fabry-Perot resonator, with the highly reflecting entrance and exit faces acting as mirrors, with the 'transmissivity' of these mirrors being dictated by the percentage area occupancy by the slits. It is relatively straightforward to take the single slit filled 
with liquid crystal discussed above and fabricate, using multiple metal slats, a much more strongly transmitting structure.

\subsubsection{With liquid crystal}

A liquid crystal filled multiple stack is constructed of blocks of 10 cells as shown in figure 8 , comprising in total a stack of 71 strips of aluminium with mylar spacers at each end.

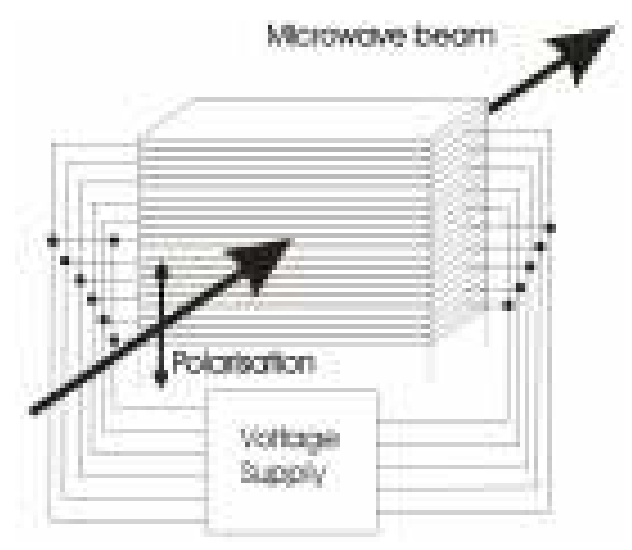

Figure 8. Part of a stack of liquid crystal filled metal slits which comprise a voltage-tuned microwave beam steering device ${ }^{21}$.

Such a structure does not just provide a selective filter ${ }^{21}$. The strong transmission resonances observed for an array of such metallic slits arises from constructive interference of the emission of radiation from the Fabry-Perot-like resonances localized in each cavity. Now, provided the frequency width of the modes is sufficient, by changing the microwave path length in separate cavities, by voltage control of the liquid crystal placed within such slits, it should be possible to control the relative phase of the emission from each cavity. Thereby for any chosen wavelength the transmission from the grating may be flexibly controlled by constructive interference of the transmission, with different intensities and phases from different slits. This will lead to the potential for low voltage beam steering.

To facilitate this microwave beam steering the 70 gaps in the structure are divided into seven groups of ten gaps in each group. An AC source $(1.0 \mathrm{kHz})$ is used to apply different voltages to each gap within one group with the gaps having the same relative position within different groups having the same voltage across them. This gives a series of seven identical sets of ten slats. The dimensions of the slats are: length $l=80.0 \mathrm{~mm}$; depth $t=30.0$ mm and metal strip thickness $d=1.0 \mathrm{~mm}$, with the width of the mylar-spaced gaps being $w=75.0 \mu \mathrm{m}$. The repeat distance of the structure is $10.75 \mathrm{~mm}$, thus the structure is actually weakly diffractive unless the same voltage is applied across every gap. Each single slit is fabricated as above with rubbed polyimide aligning a layer of E7 nematic liquid crystal. 
Only linearly polarised microwaves are incident with the electric vector lying perpendicular to the groove direction. No transmission is obtained for radiation with its E field along the slat direction. Transmission data were taken as a function of frequency with various combinations of voltages across the liquid crystal filled gaps. Voltages were now selected to give different phases of the microwave outputs at different slats thus providing, through interference, the potential for output beam steering.
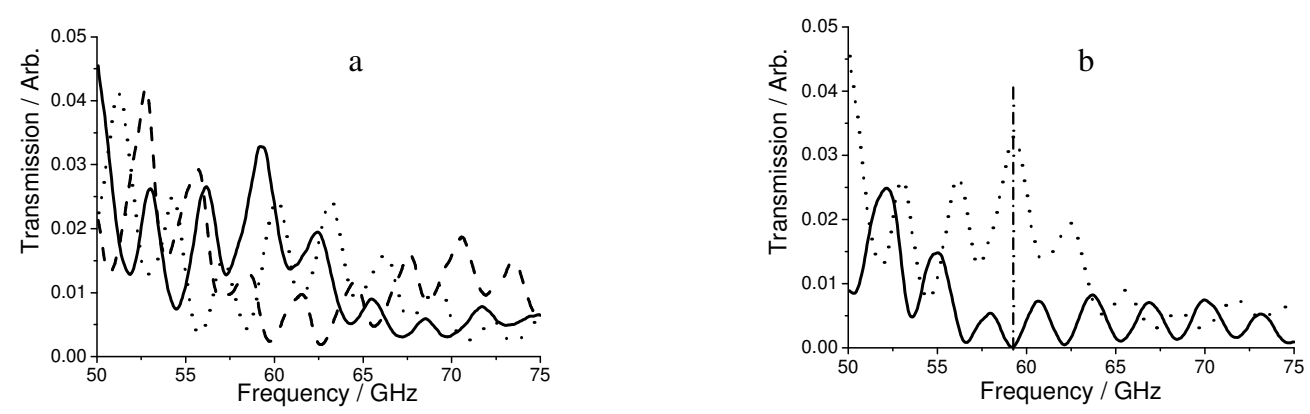

Figure 9. Transmission data as a function of frequency for different voltages applied to all cells. a. The solid line is for no voltage applied, the dotted line is for a voltage of $1.6 \mathrm{~V}_{\mathrm{rms}}$ and the dashed line is for a voltage of $3.0 \mathrm{~V}_{\mathrm{rms}}$. b. The dotted line is for no voltage applied and the solid line is for the top six gaps in each group of ten having applied voltages of $3.0 \mathrm{~V}_{\mathrm{rms}}$ with the remaining four gaps having applied voltages of $1.6 \mathrm{~V}_{\text {rms. }}$.

For the full line in figure $9 \mathrm{~b}$ the top six gaps in each group of ten have applied voltages of $3.0 \mathrm{~V}_{\text {rms }}$ with the remaining four gaps having applied voltages of $1.6 \mathrm{~V}_{\mathrm{rms}}$. This particular set of voltages was chosen to suppress the zero order transmitted beam at the selected frequency of $59.20 \mathrm{GHz}$. It is apparent that at this frequency (vertical dash-dot line in figure 9b) the signal (solid line) drops to zero while, for no applied voltage across any gap (dotted line) the signal is close to maximum. This is explained as follows. Every resonance has a width as shown in figure 9a. Across each resonant peak different frequencies will have a different phase relative to the centre, 'resonant', frequency. The frequency chosen, $59.20 \mathrm{GHz}$ is at the front edge of a resonant peak for $1.6 \mathrm{~V}_{\mathrm{rms}}$ applied, so it is approximately $-\pi / 2$ difference in phase from the central, resonant frequency at that voltage. This frequency is also at the back edge of a resonant peak for $3.0 \mathrm{~V}_{\text {rms. }}$. Thus it has approximately a $\pi / 2$ phase difference from the central resonant frequency for each gap at $3.0 \mathrm{~V}_{\mathrm{rms}}$. Since, for these two cases the two resonance peaks around $59.20 \mathrm{GHz}$ have the same order (with different index values in the cavities), the phase difference of the $59.20 \mathrm{GHz}$ signals from these two different voltage cavities will be $\sim \pi$. In addition, the relative intensity of the two resonances is $\sim 0.62(=0.0077 / 0.0123)$ comparing the $3.0 \mathrm{~V}_{\mathrm{rms}}$ with the $1.6 \mathrm{~V}_{\mathrm{rms}}$ signals shown in figure 9a. So if in every group of ten gaps a voltage of $3.0 \mathrm{~V}_{\mathrm{rms}}$ is established across the top 6 gaps and a voltage of $1.6 \mathrm{~V}_{\mathrm{rms}}$ is applied to the remaining 4 gaps, this will result in two almost equal intensity signals of opposite phase. Destructive interference of these two components results in almost zero transmission at $59.20 \mathrm{GHz}$. This clearly confirms that this structure acts as a microwave variable-phase grating. Note that with various voltages applied the grating has a pitch of $10 \mathrm{~mm}$. Thus for our working frequency higher order diffracted 
transmission signals will appear and their intensities may also be controlled by varying the distribution of voltages across the gaps.

In conclusion, by stacking a number of metallic strips with thin gaps between them and filling with aligned liquid crystal a voltage-controlled variable-phase grating at microwave frequencies is demonstrated.

\section{CONCLUSIONS}

It is clear that the use of thin metal slits filled with nematic liquid crystals is capable of yielding novel types of microwave devices. However liquid crystals still need to be developed which have low loss at microwave frequencies. Once these are available then incorporation of voltage tunable liquid crystals in other only very recently realized very thin microwave structures ${ }^{22}$ looks likely to be very interesting. Further, because the resonant wavelength of very thin slits is reduced relative to that expected from the simple Fabry-Perot condition because of the field distortion due to skin

effects then it would seem that long wavelength devices may be fabricated from very thin structures. Unfortunately there is a problem with this simplistic view. As the liquid crystal filled gaps become much less than $50 \mu \mathrm{m}$ the modes broaden quite markedly due to the losses in the metal, the finite conductivity of the metal really does matter. Of course for resonant absorbing structures this will not be a problem but for other transmissive devices such as filters and phase shifters low insertion loss will be essential.

\section{ACKNOWLEDGEMENTS}

The authors appreciate the support of QinetiQ and EPSRC and have valued discussions with Dr C R Lawrence.

\section{REFERENCES}

1. K. C. Lim, J. D. Margerum and A. M. Lackner, "Liquid crystal millimetre wave electronic phase shifter.”, Elec. Lett., 29, pp. 1065-1067, 1992.

2. K. C. Lim, J. D. Margerum, A. M. Lackner, L. J. Miller, E. Sherman and W. H. Smith, "Liquid crystal birefringence for millimetre wave radar.”, Liq. Cryst., 14, pp. 327-337, 1993.

3. F. Guerin, J. M. Chappe, P. Joffre and D. Dolfi, "Modelling, synthesis and characterization of a millimeter-wave multilayer microstrip liquid crystal phase shifter.”, Jpn. J. Appl. Phys., 36, pp. 4409-4413, 1997.

4. M. Tanaka, T. Nose and S. Sato, "Millimetre-wave transmission properties of nematic liquid-crystal cells with a grating-patterned electrode structure.”, Jpn. J. Appl. Phys., 39, pp. 6393-6396, 2000.

5. M. Tanaka and S. Sato, "Millimetre-wave deflection properties of liquid crystal prism cells with stack-layered structure.”, J. Appl. Phys., 40, pp. L1123-1125, 2002.

6. M. Tanaka and S. Sato, "Focusing properties of liquid crystal lens cells with stack-layered structure in the millimetre wave region.”, IEEE Microw. Wirel. Comp. Lett., 12, pp. 163-165, 2002.

7. H. Fujikake, T. Kuki, T. Nomoto, Y. Tsuchiya and Y. Utsumi, "Thick polymer-stabilized liquid crystal films for microwave phase control.”, J. Appl. Phys., 89, pp. 5295-5298, 2001.

8. T. Kuki, H. Fujikake and T. Nomoto, "Microwave variable delay line using dual-frequency switching-mode liquid crystal.”, IEEE Trans. Microwave Theory, 50, pp. 2604-2609, 2002. 
9. T. W. Ebbesen, H. J. Lezec, H. F. Ghaemi, T. Thio and P. A. Wolff, "Extraordinary optical transmission through sub-wavelength hole arrays.", Nature, 391, pp. 667-669, 1998.

10. L. Martin-Moreno, F. J. Garcia-Vidal, H. J. Lezec, K. M. Pellerin, T. Thio, J. B. Pendry and T. W. Ebbesen, "Theory of extraordinary optical transmission through sub-wavelength hole arrays." Phys. Rev. Lett., 86, pp.11141117,2001

11. J. A. Porto, F. J. García-Vidal and J. B. Pendry, "Transmission resonances on metallic gratings with very narrow slits.", Phys. Rev. Lett. 83, pp. 2845-2848, 1999.

12. H. E. Went, A. P. Hibbins, J. R. Sambles, C. R. Lawrence and A. P. Crick, "Selective transmission through very deep zero-order metallic gratings at microwave frequencies." Appl. Phys. Lett., 77, pp. 2789-2791, 2000.

13. A. P. Hibbins, J. R. Sambles, C. R. Lawrence and D. M. Robinson, "Remarkable transmission of microwaves through a wall of metallic bricks.” Appl. Phys. Lett., 79, pp. 2844-2846, 2001.

14. F. Yang, J. R. Sambles and G. W. Bradberry, "Long-range surface modes supported by thin films.”, Phys. Rev. B, 44, pp. 5855-5872, 1991.

15. J. R. Suckling, A. P. Hibbins, M. J. Lockyear, T. W. Preist, J. R. Sambles and C. R. Lawrence, "Finite conductance does matter for thin metal slits at microwave frequencies.” Phys. Rev. Lett., 92, pp. 147401-1-4, 2004.

16. Y. Takakura, "Optical resonance in a narrow slit in a thick metallic screen", Phys. Rev. Lett., 86, pp. 5601-5603, 2001.

17. HFSS, Ansoft Corporation, Pittsburg, U.S.A.

18. F. Yang and J. R. Sambles, "Microwave liquid crystal wavelength selector.” Appl. Phys. Lett., 79, pp. 3717-3719, 2001.

19. H. J. Deuling, "Deformation of a nematic liquid crystal in an electric field.”, Mol. Cryst. Liq. Cryst., 19, pp.123131,1972 .

20. F. Yang and J. R. Sambles, "Determination of microwave permittivities using a metallic slit.” J. Phys. D: Appl. Phys., 35, pp. 3049-3051, 2002.

21. F. Yang and J. R. Sambles, "Microwave liquid crystal variable phase grating." Accepted for Appl. Phys. Lett., 2004.

22. A. P. Hibbins, J. R. Sambles, C. R. Lawrence and J. R. Brown, "Squeezing millimetre waves into microns." Phys. Rev. Lett., 92, pp. 143904-1-4, 2004 